\section{IDDF2018-ABS-0065 EFFICACY AND EFFECTIVENESS EVALUATION OF ENDOSCOPIC FOR STOMACH CANCER SCREENING, COMPARED TO RADIOGRAPHY AND PEPSINOGEN TEST IN HIGH RISK PEOPLE IN IRAN}

Fatemeh Karami* ${ }^{*}$ Tehran University of Medical Science, Iran

10.1136/gutjnl-2018-IDDFabstracts.4

Background Gastric cancer is the fourth most common cancer and the second cause of cancer death throughout the world the prevalence of gastric cancer (GC) progressively increased during the last decades in Iran. Health-care systems are always faced with high costs of treatment of gastrointestinal cancers including stomach cancer. The best strategy to reduce the mortality for GC is to schedule appropriate screening and surveillance programs, which raises many relevant concerns taking into account its worldwide variability, natural history, diagnostic tools, therapeutic strategies, and cost-effectiveness.

The aim of this study was to evaluate the effectiveness and cost-effectiveness of gastric cancer screening methods (Endoscopy, Radiography and Pepsinogen testing) comparing with themselves and with no screening in high risk people in Iran.

Methods A systematic review of relevant databases, including PubMed, Cochran, Scopus, Google Scholar was conducted. The evaluating the quality of studies were carried out independently by two persons using standard checklists. To assess the cost-effectiveness, the model of decision tree was used. The hypothetical population of 1 Million of the high risk of GC were entered into the model. The study was undertaken from healthcare payer perspective. The outcome of the study was the 5 years survival and QALY of the high risk people.

Results The 2866 studies were found in the first phase of searching through databases. After eliminating duplicate studies and studies incompatible with the inclusion and exclusion criteria of our study, 8 studies selected. The sensitivity of Endoscopy, radiography and pepsinogen testing were 93\%,83\% and $77 \%$ respectively. The result of modelling showed that with no screening, only 984875 persons out of 1 million high risk individuals would survive after 5 years; whereas by Endoscopy and Radiography and pepsinogen testing 999,691, 999344 and 999113 people will have 5 years survival, respectively.

Conclusions In terms of effectiveness, endoscopy is more susceptible to both radiographic tests and pepsinogen tests. The cost of treatment in the non-screening program was estimated at three times the endoscopic screening program. Given the high effectiveness of endoscopy and its therapeutic aspect, it is the best option for screening the population at risk.

\section{IDDF2018-ABS-0069 HELICOBACTER PYLORI DNA PROMOTES CELLULAR PROLIFERATION, MIGRATION AND INVASION OF GASTRIC CANCER BY ACTIVATING TOLL-LIKE RECEPTOR 9}

Xiaoyong Wang*, Jin Huang. Department of Gastroenterology, Changzhou No. 2 People's Hospital, Affiliated with Nanjing Medical University, China

\subsection{6/gutjnl-2018-IDDFabstracts.5}

Background Helicobacter pylori (H. pylori) infection is a wellknown risk factor for gastric cancer. Toll-like receptor 9 (TLR9) plays an important role in many cancers and is important for immunity to $H$. pylori infection. Thus, this study aimed to evaluate the influence of $H$. pylori on TLR9 and explore its roles in gastric cancer.

Methods TLR9 expression in MKN45 cells was detected using qRT-PCR and western blot assays. Then, TLR9 was knocked down by RNA interference (RNAi) technology in MKN45 cells. The CCK-8 assay was performed to investigate cell proliferation, and the Transwell system was established to test the migrative and invasive abilities of MKN45 cells.

Results $H$. pylori infection or H. pylori DNA level was positively correlated with TLR9 upregulation in MKN45 cells. In vitro, $H$. pylori DNA significantly accelerated cell proliferation and promoted the migration and invasion in MKN45 cells. In contrast, the knockdown of TLR9 significantly suppressed cell proliferation and inhibited the migration and invasion in MKN45 cells.

Conclusions The present results suggest that the $H$. pylori DNA/TLR9 signalling pathway plays an important role in gastric cancer, which might be a potent therapeutic target.

\section{IDDF2018-ABS-0072 IDENTIFICATION OF HUB GENES AND ANALYSIS OF PROGNOSTIC VALUES IN PANCREATIC DUCTAL ADENOCARCINOMA BY INTEGRATED BIOINFORMATICS METHODS}

Yi Lu*, Chu-Jun Li, Hong-Lei Chen, Jia-Chen Sun. Department of Gastrointestinal Endoscopy, The Sixth Affiliated Hospital of Sun Yat-sen University, China

\subsection{6/gutjnl-2018-IDDFabstracts.6}

Background Pancreatic ductal adenocarcinoma (PDAC) is one of the lethal cancers in the world, and more molecular mechanisms should be illuminated to meet the urgent need of developing novel detection and therapeutic strategies. We analysed the related microarray data to find the possible hub genes and analysed their prognostic values using bioinformatics methods.

Methods The mRNA microarray datasets GSE62452, GSE15471, GSE102238, GSE16515, and GSE62165, were finally chosen and analysed using GEO2R. The overlapping genes were found by Venn Diagrams, functional and pathway enrichment analyses were performed using the DAVID database, and the protein-protein interaction (PPI) network was constructed by STRING and Cytoscape. OncoLnc, which linked TCGA survival data was used to investigate the prognostic values.

Results In total, 179 differentially expressed genes (DEGs) were found in PDAC, among which, 130 were up-regulated genes, and 49 were down-regulated. DAVID showed that the up-regulated genes were significantly enriched in extracellular matrix and structure organisation, collagen catabolic and metabolic process, while the down-regulated genes were mainly involved in proteolysis, reactive oxygen species metabolic process, homeostatic process and cellular response to starvation. From the PPI network, the 22 nodes with the highest degree were screened as hub genes. Based on Molecular Complex Detection (MCODE) plug-in, the top module was formed by EGF, PLAT, MMP7, ALB, TIMP1, F8, PLAU, LAM3, MMP1, ITGA2, LAMB3 and LAMC2. OncoLnc survival analysis showed that high expression of ITGA2, MMP7, ITGB4, ITGA3, VCAN and PLAU might predict poor survival results in PDAC.

Conclusions The present study identified hub genes and pathways in PDAC, which may be a potential target for its diagnosis, treatment, and prognostic prediction. 